\title{
Adsorption of Metals from FAME of Nigerian Spent Vegetable Oils Using Waste Printing Paper: Non-Food Option for Biodiesel to Safe Food Security and Environment
} (Part II)

\author{
*MOYIB, OK; OMOTOLA, OE
}

\author{
Department of Chemical Sciences, Tai Solarin University of Education, Ijagun. PMB 2118, Ijebu-Ode Nigeria \\ *Corresponding Author: kmoyib@hotmail.com; moyibok@tasued.edu.ng; \\ Tel: $+234(0) 7019595255,09063491211$
}

\begin{abstract}
Fatty acid methyl ester (FAME) from spent vegetable oils requires pre and or post-transesterification treatment to meet International norms for biodiesel. The present study assessed the potential of waste printing paper (WPP) as post-transesterification treatment for adsorption of metals from FAME obtained from Nigerian neat and spent vegetable oils (NSpVOs). The WPP adsorbent was prepared according to Moyib et al. (2017). The adsorption experiments for the removal of single metal ions were carried out using $10 \mathrm{~mL}$ FAME samples, $1.0 \mathrm{~g}$ WPP at a constant $25^{\circ} \mathrm{C}, \mathrm{pH} 6$ for $60 \mathrm{~min}$. At the end of the contact time, alkali metals in FAMEs were estimated according to EN 14108 and EN 14109 for $\mathrm{Na}$ and $\mathrm{K}$, respectively and alkaline and heavy metals followed AOAC (2005). The results revealed WPP was able to reduce the metal content in the FAME to acceptable limit level and also, enhanced biofuel properties such as acid value (AV) and Conradson carbon (CC) but with loss of minute FAME. Cluster analysis distinguished FAMEs after and before WPP adsorption treatment. Part I and II of this study show feasibility for diversifying Nigerian spent vegetable oils as non-food option for production of biodiesel and achieve low carbon footprint. Also, WPP as locally available and cheap adsorbent showed good potential as post-transesterification treatment of FAME to desirable quality.
\end{abstract}

\section{DOI: https://dx.doi.org/10.4314/jasem.v22i5.36}

Copyright: Copyright $\odot 2018$ Moyib and Omotola. This is an open access article distributed under the Creative Commons Attribution License (CCL), which permits unrestricted use, distribution, and reproduction in any medium, provided the original work is properly cited.

Dates: Received: 09 January, 2018, Revised: 02 May 2018, Accepted: 05 May 2018

Key words: Adsorption, fatty acid methyl ester, metals, spent Nigerian vegetable oils, waste printing paper

Spent vegetable oils (SpVOs) are cooking oils that have undergone several cooking process, presented with altered physicochemical properties and are no longer useful for human consumption due to formation of oxidation products that are detrimental to human health (Felizardo et al., 2006). Therefore, they are directed for other uses other than human consumption or discarded. Various rechanneling of SpVOs include as food for beef cattle and raw material for production of soap. Traditionally, SpVOs are indiscriminately discharged into sewage or used as fuel in burning and wood cooking, resulting into soil and water pollution and increased bioremediation costs. They have been successful used for production of biodiesel in USA, Canada, Brazil, China, Austria and Germany (Leung and Chen, 2000; Felizardo et al., 2006; Canakci, 2007; de Araujo et al., 2013; Filho et al., 2014). Proportion of biodiesel generating from SpVOs, though, may not be sufficient but could provide significant proportion of world biodiesel production and complement biodiesel as blending agent to lower reliance on food crops and overall cost (Cvengroš and Cvengrošová, 2004; Canakci, 2007). In addition, diversifying SpVOs into biofuel production has a potential in reducing environmental impacts of $\mathrm{GHG}$ and $\mathrm{CO}_{2}$ emission, translating into safe and healthy environment (Felizardo et al., 2006; Bart et al., 2010). Chemical analyses showed SpVOs constitute heterogenous mixture and therefore, required pre-transesterification treatment steps such as filtering, vacuum distilling, solvent extraction, adsorption filtration and acidesterification step. Also, some post-transesterification treatments such as adsorption using silica gel beads, activated carbon, bleaching earth have been reported (Encinar et al., 2005; Kheang et al., 2006; de Araujo et al., 2013; Asri et al., 2015). Two steps acid-base and alkali transesterification have been successfully used to transform WVOs and SpVOs into good quality FAME (Bart et al., 2010; Bakir and Fadhil; 2011; de Araujo et al., 2013; Personal data). Whatsoever, many of these studies didn't assess effect of treatments on metallic content of FAME and FAME with combined group I and II above 5 ppm content is not considered biodiesel by International standards. A previous study as Part I of the present study by the same researchers revealed that two-steps alkali transesterification is efficient in obtaining FAME with good biofuel properties from NSpVOs but not sufficient for total

*Corresponding Author: kmoyib@hotmail.com; moyibok@tasued.edu.ng; Tel: +234(0)7019595255, 
removal of metals from FAME and thus a posttransestrfication treatment, perhaps, adsorption process might be necessary to accomplish this (In press). In addition, some post-transesterification treatments employed involve used of imported materials and toxic substances that may constitutes further environmental hazards. Adsorption of metals treatment using locally available biomaterials such as waste printing paper (WPP), perhaps, could be a bio economic and environmental friendly viable option for Nigeria to gain carbon neutral (Moyib et al., 2017). Therefore, at present, this study sought the potential of WPP for adsorption of metals from FAME produced from fresh vegetable oils and NSpVOs as a posttreatment following two steps alkali esterification that is environmental friendly and economic viable.

\section{MATERIALS AND METHODS}

WPP adsorbent preparation and adsorption experiments for metals removals: WPP adsorbent was prepared according to Moyib et al. (2017). The adsorption experiments for the removal of single metal ions from the previously prepared FAME from fresh VOs ((Nigerian palm methyl ester, NPME; Nigerian peanut methyl ester, NPeME) and NSpVOs (Nigerian spent palm methyl ester, NSPME; and Nigerian spent peanut methyl ester, NSPeME) were carried out in triplicates with $10 \mathrm{~mL}$ FAME using $1.0 \mathrm{~g}$ WPP at a constant temperature of $25^{\circ} \mathrm{C}, \mathrm{pH} 6$ for 60 min with constant shaking using an electric shaker (Moyib et al., 2017). At the end of the contact time, the mixtures were filtered using Whatman no. 1 filter paper and left for 30 min to allow last dropping of FAME and final volume was recorded. Alkali metals were estimated using the sulphated ash according to EN 14108 and EN 14109 for $\mathrm{Na}$ and $\mathrm{K}$, respectively and alkaline metals and heavy metals followed AOAC (2005) using Atomic Absorption Spectrometer (AAS) single hollow cathode lamp (Buck Scientific, USA).

Data Management: Adsorption efficacy (AE, \% $\mathrm{mg} / \mathrm{L}$ ); metal uptake (Qt, $\mathrm{mg} / \mathrm{g}$ ), the concentration of metal adsorbed per unit mass of adsorbent at time, $t$; and metal adsorbed (Qe, $\mathrm{mg} / \mathrm{g}$ ), the amount of metal adsorbed per unit mass of adsorbent at equilibrium were also according to Moyib et al. (2017) and Freundlich adsorption isotherm model fitness was used for evaluation of adsorption process by WPP. All data analyses for descriptive, ANOVA, and correlation were carried out using SAS version 9.2 (SAS, 2002) and principal component analysis for visual clustering and classification of FAME before and after adsorption with WPP was by Dissimilarity representative for Windows (Darwin, Perrier and Jacquemoud-Collet, 2006).

\section{RESULTS AND DISCUSSION}

Adsorption efficacy of WPP for metals removal in FAME: Tables 1 and 2 show the adsorption properties of WPP for alkali, alkaline and heavy metal ions, $\mathrm{Na}^{+}$, $\mathrm{K}^{+}, \mathrm{Mg}^{2+}, \mathrm{Ca}^{2+}, \mathrm{Al}^{3+}, \mathrm{Fe}^{+2}, \mathrm{~Pb}^{2+}$, and $\mathrm{Cu}^{+2}$ following two steps alkali transesterification process. The lowest and highest AE of WPP was observed for $\mathrm{Na}(66.6 \%$ in NPeME) and $\mathrm{Fe}(80.65 \%$, in NSPeME), respectively. Adsorbed metal per unit mass of WPP at equilibrium (qe) ranged from $0.0004 \mathrm{mg} / \mathrm{g}$ for $\mathrm{Pb}$ from NPeME to $0.119 \mathrm{mg} / \mathrm{g}$ for $\mathrm{Na}$ from NSPeME. The lowest and highest concentration of metal remained in final FAME (Cf) was $0.014 \mathrm{mg} / \mathrm{L}$ for $\mathrm{Pb}$ in NPeME and $4.21 \mathrm{mg} / \mathrm{L}$ for $\mathrm{Na}$ in NSPeME. Therefore, WPP was able to reduce the metal content of the generated FAME from neat VOs and NSPVOs. Table 3 shows the biofuel properties and metallic content of the finalized FAME after post transesterification treatment with WPP following two-steps transesterification process were within International norms across the globe. Comparing Table 3 in the present study to Figure 3 in Part I study shows loss of some FAME to the adsorbent (about $0.5 \%$ ) and pronounced reduction in biofuel properties such as density, AV, CC and water and sediment. The present results is comparable to results of Kheang et al. (2006) using various adsorbent for obtaining good quality biodiesel from frying oil.

Freundlich Adsorption isotherms fitness: Figure 1 and 2 reveal Freundlich adsorption isotherms slope greater than unity $(1 / n>1)$ for the presented metals except $\mathrm{Fe}^{2+}$ and therefore, $\mathrm{n}$ is less than unity $(\mathrm{n}<1)$ and the intercept $\log \mathrm{Kf}$ have negative integers, indicating unfavorable adsorption process and low adsorption capacity of WPP for $\mathrm{Na}^{+}, \mathrm{K}^{+}, \mathrm{Mg}^{2+}, \mathrm{Ca}^{2+}, \mathrm{Al}^{3+}, \mathrm{Pb}^{2+}$, and $\mathrm{Cu}^{2+}$ in FAME. The present adsorption isotherm values of WPP for removal of these metals from FAME are lower when compared to some prepared and derived adsorbents for similar roles in synthetic and natural waste waters (Roh et al., 2014; Moyib et $a l ., 2017)$. Nevertheless, the adsorption intensity of $(n<1)$ obtained at present is an indicative of S-type isotherm that is cooperative in nature and usually observed at low concentration ranges for compounds containing polar functional groups that are in constant competition for adsorption sites (Kumar and Bandyopadhyay, 2006). The observed unfavorable adsorption process and low capacity of WPP for these metals at present indicate heterogeneous nature of FAME constituting polar compounds such as fatty acid esters, methyl esters, and oxidized organic products competing with these metals for adsorption sites on WPP. 
Table 1: Descriptive statistics for adsorption of alkali and alkaline metals from NPME, NPeME, NSPME, NSPeME by WPP as posttransesterification treatment.

\begin{tabular}{|c|c|c|c|c|c|c|c|c|c|}
\hline & $\begin{array}{l}\text { WPP } \\
\text { Adsorption } \\
\text { properties }\end{array}$ & Mean & SD & SE & $\mathrm{CV}$ & Range & Min & Max & $\overline{\mathbf{N}}$ \\
\hline & NPME & & & & & & & & \\
\hline \multirow[t]{3}{*}{$\mathbf{N a}$} & $\mathrm{Cf}$ & 2.233 & 0.153 & 0.088 & 6.84 & 0.3 & 2.1 & 2.4 & 3 \\
\hline & $\mathrm{AE}$ & 67.167 & 0.491 & 0.283 & 0.73 & 0.98 & 66.667 & 67.647 & 3 \\
\hline & Qt & 0.043 & 0.002 & 0.001 & 5.162 & 0.004 & 0.04 & $0.045 \dagger$ & 3 \\
\hline \multirow[t]{3}{*}{$\mathbf{K}$} & $\mathrm{Cf}$ & 0.437 & 0.051 & 0.03 & 11.752 & 0.1 & 0.38 & 0.48 & 3 \\
\hline & $\mathrm{AE}$ & 70.288 & 0.104 & 0.06 & 0.148 & 0.203 & 70.173 & 70.375 & 3 \\
\hline & Qt & 0.01 & 0.001 & 0.001 & 13.121 & 0.002 & 0.008 & $0.011 \dagger$ & 3 \\
\hline \multirow[t]{3}{*}{ Mg } & $\mathrm{Cf}$ & 0.424 & 0.032 & 0.018 & 7.488 & 0.063 & 0.39 & 0.453 & 3 \\
\hline & $\mathrm{AE}$ & 75.062 & 0.059 & 0.034 & 0.079 & 0.114 & 75.014 & 75.128 & 3 \\
\hline & Qt & 0.012 & 0.001 & 0 & 7.166 & 0.002 & 0.011 & $0.013 \dagger$ & 3 \\
\hline \multirow[t]{4}{*}{$\mathrm{Ca}$} & $\mathrm{Cf}$ & 0.255 & 0.044 & 0.025 & 17.18 & 0.085 & 0.218 & 0.303 & 3 \\
\hline & $\mathrm{AE}$ & 73.003 & 0.004 & 0.002 & 0.006 & 0.007 & 73.001 & 73.008 & 3 \\
\hline & $\mathrm{Qt}$ & 0.007 & 0.001 & 0.001 & 18.178 & 0.002 & 0.005 & $0.008 \dagger$ & 3 \\
\hline & NPeME & & & & & & & & \\
\hline \multirow[t]{3}{*}{$\mathrm{Na}$} & $\mathrm{Cf}$ & 1.29 & 0.026 & 0.015 & 2.051 & 0.05 & 1.27 & 1.32 & 3 \\
\hline & $\mathrm{AE}$ & 66.947 & 0.322 & 0.186 & 0.481 & 0.601 & 66.579 & 67.179 & 3 \\
\hline & Qt & 0.024 & 0.001 & 0 & 3.37 & 0.001 & 0.024 & $0.025 \dagger$ & 3 \\
\hline \multirow[t]{3}{*}{$\mathbf{K}$} & $\mathrm{Cf}$ & 0.603 & 0.06 & 0.035 & 9.991 & 0.12 & 0.54 & 0.66 & 3 \\
\hline & $\mathrm{AE}$ & 70.214 & 0.146 & 0.084 & 0.208 & 0.292 & 70.068 & 70.36 & 3 \\
\hline & $\mathrm{Qt}$ & 0.013 & 0.001 & 0.001 & 8.683 & 0.002 & 0.012 & $0.015 \dagger$ & 3 \\
\hline \multirow[t]{3}{*}{ Mg } & $\mathrm{Cf}$ & 0.17 & 0.05 & 0.029 & 29.412 & 0.1 & 0.12 & 0.22 & 3 \\
\hline & $\mathrm{AE}$ & 75.262 & 0.23 & 0.133 & 0.305 & 0.454 & 75.057 & 75.51 & 3 \\
\hline & Qt & 0.005 & 0.001 & 0.001 & 29.974 & 0.003 & 0.003 & $0.006 \dagger$ & 3 \\
\hline \multirow[t]{4}{*}{$\mathrm{Ca}$} & $\mathrm{Cf}$ & 0.097 & 0.036 & 0.021 & 37.503 & 0.072 & 0.061 & 0.133 & 3 \\
\hline & $\mathrm{AE}$ & 73.111 & 0.139 & 0.081 & 0.191 & 0.267 & 73.001 & 73.268 & 3 \\
\hline & $\mathrm{Qt}$ & 0.002 & 0.001 & 0.001 & 36.003 & 0.002 & 0.002 & $0.003 \dagger$ & 3 \\
\hline & NSPME & & & & & & & & \\
\hline \multirow[t]{3}{*}{$\mathrm{Na}$} & $\mathrm{Cf}$ & 4.063 & 0.074 & 0.043 & 1.456 & 0.14 & 4.08 & 4.12 & 3 \\
\hline & $\mathrm{AE}$ & 72.845 & 1.179 & 0.681 & 1.713 & 2.358 & 70.683 & 73.041 & 3 \\
\hline & $\mathrm{Qt}$ & 0.115 & 0.008 & 0.005 & 7.477 & 0.016 & 0.113 & $0.117 \dagger$ & 3 \\
\hline \multirow[t]{3}{*}{$\mathbf{K}$} & $\mathrm{Cf}$ & 0.73 & 0.15 & 0.087 & 20.548 & 0.3 & 0.58 & 0.88 & 3 \\
\hline & $\mathrm{AE}$ & 70.227 & 0.171 & 0.099 & 0.244 & 0.34 & 70.068 & 70.408 & 3 \\
\hline & Qt & 0.016 & 0.003 & 0.002 & 20.316 & 0.007 & 0.013 & $0.02 \dagger$ & 3 \\
\hline \multirow[t]{3}{*}{ Mg } & $\mathrm{Cf}$ & 0.773 & 0.189 & 0.109 & 24.41 & 0.37 & 0.61 & 0.98 & 3 \\
\hline & $\mathrm{AE}$ & 75.091 & 0.086 & 0.049 & 0.114 & 0.17 & 75 & 75.17 & 3 \\
\hline & Qt & 0.022 & 0.005 & 0.003 & 22.962 & 0.01 & 0.018 & $0.028 \dagger$ & 3 \\
\hline \multirow[t]{4}{*}{$\mathrm{Ca}$} & $\mathrm{Cf}$ & 0.291 & 0.056 & 0.032 & 19.107 & 0.109 & 0.23 & 0.339 & 3 \\
\hline & $\mathrm{AE}$ & 73.021 & 0.018 & 0.01 & 0.024 & 0.033 & 73.001 & 73.033 & 3 \\
\hline & Qt & 0.008 & 0.001 & 0.001 & 19.841 & 0.003 & 0.006 & $0.009 \dagger$ & 3 \\
\hline & NSPeME & & & & & & & & \\
\hline \multirow[t]{3}{*}{$\mathrm{Na}$} & $\mathrm{Cf}$ & 4.083 & 0.114 & 0.066 & 2.237 & 0.22 & 4.09 & 4.21 & 3 \\
\hline & $\mathrm{AE}$ & 72.033 & 1.97 & 1.137 & 2.854 & 3.863 & 71.879 & 72.742 & 3 \\
\hline & $\mathrm{Qt}$ & 0.116 & 0.007 & 0.004 & 6.426 & 0.014 & 0.114 & $0.119 \dagger$ & 3 \\
\hline \multirow[t]{3}{*}{$\mathbf{K}$} & $\mathrm{Cf}$ & 0.73 & 0.15 & 0.087 & 20.548 & 0.3 & 0.58 & 0.88 & 3 \\
\hline & $\mathrm{AE}$ & 70.227 & 0.171 & 0.099 & 0.244 & 0.34 & 70.068 & 70.408 & 3 \\
\hline & Qt & 0.016 & 0.003 & 0.002 & 19.216 & 0.006 & 0.013 & $0.019 \dagger$ & 3 \\
\hline \multirow[t]{3}{*}{ Mg } & $\mathrm{Cf}$ & 0.773 & 0.254 & 0.147 & 32.824 & 0.49 & 0.49 & 0.98 & 3 \\
\hline & $\mathrm{AE}$ & 75.073 & 0.126 & 0.073 & 0.168 & 0.219 & 75 & 75.219 & 3 \\
\hline & Qt & 0.022 & 0.007 & 0.004 & 32.828 & 0.014 & 0.014 & $0.028 \dagger$ & 3 \\
\hline \multirow[t]{3}{*}{$\mathrm{Ca}$} & $\mathrm{Cf}$ & 0.268 & 0.148 & 0.086 & 55.298 & 0.285 & 0.102 & 0.387 & 3 \\
\hline & $\mathrm{AE}$ & 73.073 & 0.071 & 0.041 & 0.097 & 0.139 & 73.011 & 73.15 & 3 \\
\hline & Qt & 0.007 & 0.004 & 0.002 & 55.516 & 0.007 & 0.003 & $0.01 \dagger$ & 3 \\
\hline
\end{tabular}

$\dagger Q e$, amount of metal adsorbed per unit mass of WPP at equilibrium, Cf, final concentration of metal in FAME, AE, adsorption efficacy of WPP for metal, Qt amount of adsorbate adsorbed by WPP from diesel at time $t$. 
Table 2: Descriptive statistics for adsorption of aluminium and some heavy metals NPME, NPeME, NSPME, NSPeME by WPP as posttransesterification treatment.

\begin{tabular}{|c|c|c|c|c|c|c|c|c|c|}
\hline & $\begin{array}{l}\text { Adsorption } \\
\text { properties }\end{array}$ & Mean & SD & $\mathrm{SE}$ & $\mathrm{CV}$ & Range & Min & $\operatorname{Max}$ & $\mathrm{N}$ \\
\hline & NPME & & & & & & & & \\
\hline \multirow[t]{3}{*}{ Al } & Cf & 0.561 & 0.083 & 0.048 & 14.821 & 0.166 & 0.48 & 0.646 & 3 \\
\hline & $\mathrm{AE}$ & 80.023 & 0.01 & 0.006 & 0.013 & 0.021 & 80.013 & 80.033 & 3 \\
\hline & $Q t$ & 0.021 & 0.003 & 0.002 & 14.549 & 0.006 & 0.018 & 0.024 & 3 \\
\hline \multirow[t]{3}{*}{$\mathrm{Fe}$} & Cf & 0.129 & 0.012 & 0.007 & 9.543 & 0.024 & 0.119 & 0.143 & 3 \\
\hline & $\mathrm{AE}$ & 80.095 & 0.105 & 0.061 & 0.131 & 0.208 & 80 & 80.208 & 3 \\
\hline & $Q t$ & 0.005 & 0.001 & 0 & 11.714 & 0.001 & 0.004 & 0.006 & 3 \\
\hline \multirow[t]{3}{*}{$\mathrm{Pb}$} & Cf & 0.112 & 0.042 & 0.024 & 37.478 & 0.084 & 0.07 & 0.155 & 3 \\
\hline & $\mathrm{AE}$ & 75.006 & 0.007 & 0.004 & 0.01 & 0.014 & 75 & 75.014 & 3 \\
\hline & $Q t$ & 0.003 & 0.001 & 0.001 & 39.048 & 0.003 & 0.002 & 0.005 & 3 \\
\hline \multirow[t]{3}{*}{$\mathrm{Cu}$} & Cf & 0.582 & 0.103 & 0.059 & 17.675 & 0.194 & 0.465 & 0.659 & 3 \\
\hline & $\mathrm{AE}$ & 77.022 & 0.019 & 0.011 & 0.025 & 0.037 & 77 & 77.037 & 3 \\
\hline & $\begin{array}{l}\text { Qt } \\
\text { NPeME }\end{array}$ & 0.019 & 0.003 & 0.002 & 18.006 & 0.006 & 0.015 & 0.021 & 3 \\
\hline \multirow[t]{3}{*}{ Al } & $\mathrm{Cf}$ & 0.035 & 0.009 & 0.005 & 24.232 & 0.017 & 0.027 & 0.044 & 3 \\
\hline & $\mathrm{AE}$ & 80.31 & 0.253 & 0.146 & 0.315 & 0.483 & 80.025 & 80.508 & 3 \\
\hline & Qt & 0.001 & 0 & 0 & 24.917 & 0.001 & 0.001 & 0.002 & 3 \\
\hline \multirow[t]{3}{*}{$\mathrm{Fe}$} & Cf & 0.124 & 0.021 & 0.012 & 16.685 & 0.041 & 0.102 & 0.143 & 3 \\
\hline & $\mathrm{AE}$ & 80.043 & 0.04 & 0.023 & 0.049 & 0.078 & 80 & 80.078 & 3 \\
\hline & $Q t$ & 0.005 & 0.001 & 0 & 15.9 & 0.001 & 0.004 & 0.005 & 3 \\
\hline \multirow[t]{3}{*}{$\mathrm{Pb}$} & Cf & 0.042 & 0.028 & 0.016 & 66.8 & 0.0 & 0.014 & 0.07 & 3 \\
\hline & $\mathrm{AE}$ & 75.0 & 0.053 & 0.031 & & 0.1 & 75.004 & 75.111 & 3 \\
\hline & $Q t$ & 0.001 & 0.001 & 0 & 67.236 & 0.002 & 0.0004 & 0.002 & 3 \\
\hline \multirow[t]{3}{*}{$\mathrm{Cu}$} & $\mathrm{Cf}$ & 0.185 & 0.032 & 0.019 & 17.398 & 0.064 & 0.155 & 0.219 & 3 \\
\hline & $\mathrm{AE}$ & 77. & 0.0 & 0. & & 0.0 & 77.016 & 77.098 & 3 \\
\hline & $\begin{array}{l}\mathrm{Qt} \\
\mathrm{NSPME}\end{array}$ & 0.006 & 0.001 & 0.001 & 17. & 0.002 & 0.005 & 0. & 3 \\
\hline \multirow[t]{3}{*}{ A1 } & $\mathrm{Cf}$ & 0.561 & 0.083 & 0.048 & 14.821 & 0.166 & 0.48 & 0.646 & 3 \\
\hline & $\mathrm{AE}$ & 80 & & & & & 80. & & 3 \\
\hline & $Q t$ & & 0. & 0.002 & & 6 & & & 3 \\
\hline \multirow[t]{3}{*}{$\mathrm{Fe}$} & Cf & 0.129 & 0.012 & 0.007 & & 0.024 & 0.119 & & 3 \\
\hline & $\mathrm{AE}$ & 80.057 & 0.054 & 0.031 & 0.067 & 0.107 & 80.007 & 80.114 & 3 \\
\hline & $Q t$ & & 0.0 & 0 & & & 0. & & 3 \\
\hline \multirow[t]{3}{*}{$\mathrm{Pb}$} & Cf & 0.112 & 0.042 & 0.024 & 37.478 & 0.0 & 0.07 & 0.155 & 3 \\
\hline & $\mathrm{AE}$ & 75.006 & 0.007 & 0.004 & 0. & 0.0 & 75 & 75.014 & 3 \\
\hline & Qt & 0.003 & 0.001 & 0.001 & 39.048 & 0.003 & 0.002 & 0.005 & 3 \\
\hline \multirow[t]{3}{*}{$\mathrm{Cu}$} & Cf & 0.582 & 0.103 & 0.059 & 17.675 & 0.194 & 0.465 & 0.659 & 3 \\
\hline & $\mathrm{AE}$ & 77022 & 0.019 & & & & 77 & 37 & 3 \\
\hline & $\begin{array}{l}\text { Qt } \\
\text { NSPeME }\end{array}$ & 0.019 & 0.003 & 0.002 & 18.006 & 0.006 & 0.015 & 0.021 & 3 \\
\hline \multirow[t]{3}{*}{ Al } & $\mathrm{Cf}$ & 0.40 & 0.156 & 0.09 & 38. & 0.2 & 0.224 & 0.502 & 3 \\
\hline & $\mathrm{AE}$ & 80.031 & 0.003 & 0.002 & & & 80.028 & & 3 \\
\hline & Qt & 0.015 & 0.006 & 0.003 & 38.706 & 0.011 & 0.008 & 0.019 & 3 \\
\hline \multirow[t]{3}{*}{$\mathrm{Fe}$} & $\mathrm{Cf}$ & 0.293 & 0.085 & 0.049 & & & 0.23 & & 3 \\
\hline & $\mathrm{AE}$ & 80.34 & 0.31 & 0179 & & 0.62 & 80.035 & $0<=5$ & 3 \\
\hline & Qt & & 0.003 & 0.0 & 29. & 0. & 0.009 & & 3 \\
\hline \multirow[t]{3}{*}{$\mathrm{Pb}$} & Cf & & 0.032 & 0.019 & 35. & 0.056 & 0.07 & 0.127 & 3 \\
\hline & $\mathrm{AE}$ & 75.0 & & & & & 75 & & 3 \\
\hline & Qt & 0.003 & 0.001 & 0.001 & 35.932 & & 0.002 & 0.004 & 3 \\
\hline \multirow[t]{3}{*}{$\mathrm{Cu}$} & Cf & 0.433 & 0.386 & 0.223 & 89.013 & 0.769 & 0.033 & 0.802 & 3 \\
\hline & $\mathrm{AE}$ & 77.0 & 0.017 & 0.01 & 0. & 0.0 & 77.004 & 77.037 & 3 \\
\hline & Qt & 0.014 & 0.012 & 0.007 & 89.101 & 0.024 & 0.001 & 0.025 & 3 \\
\hline
\end{tabular}

SA, South Africa, Nig., Nigeria

Therefore, the present adsorption of FAME is considered as heterogeneous adsorption of multiple polar species (Proctor and Toro Vanzquez, 2009). This is obviously supported with reduced values for AV and $\mathrm{CC}$ in FAMEs at post transeterification treatment with WPP as presented in Table 3. In addition, metal adsorption in synthetic waste water are studied at varying higher concentration of metals ranging between 50 and $500 \mathrm{ppm}$ and some adsorbents are chemically modified to increase absorptivity of the adsorbates (metals) and efficiency of adsorbents (Moyib et al., 2017; Roh et al., 2014). 
Table 3: Biofuel properties of finalized NPME, NPeME, NSPME and NSPeM at post-two steps transesterification treatment with WPP in comparison with International standards for FAME and Alkyl esters

\begin{tabular}{|c|c|c|c|c|c|c|c|c|c|}
\hline FAME & NPME & AReME & NSPME & NSPeME & EN 14214 & $\begin{array}{l}\text { ASTM } \\
\text { D6751 }\end{array}$ & Australian & Indian & SA \\
\hline Diesel \% xal & 82.04 & 90.56 & 80.07 & 80.04 & - & 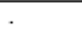 & $\cdot$ & 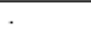 & - \\
\hline Glycerol \% xol & 9.43 & 8.67 & 9.71 & 9.75 & 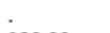 & . & . & - & . \\
\hline $\begin{array}{l}\text { FP }{ }^{\circ} \mathrm{C} \\
\text { Density } 15^{\circ} \mathrm{C} \mathrm{g} / \mathrm{cm}^{3}\end{array}$ & $\begin{array}{l}174.33 \\
0.863\end{array}$ & $\begin{array}{l}174.67 \\
0.861\end{array}$ & $\begin{array}{l}187.33 \\
0.869\end{array}$ & $\begin{array}{l}185.00 \\
0.871\end{array}$ & $\begin{array}{l}120.00 \\
0.86-0.90\end{array}$ & 130.00 & $\begin{array}{l}120.00 \\
0.86-0.9\end{array}$ & $\begin{array}{l}120.00 \\
0.86-0.9\end{array}$ & $\begin{array}{l}120.00 \\
0.86-0.1\end{array}$ \\
\hline & 0.863 & 0.86 & 0.873 & 0.88 & & & & & \\
\hline Viscosity $40^{\circ} \mathrm{C} \mathrm{mm2/s}$ & 3.89 & 3.77 & 4.47 & 4.49 & $3.5-5$ & $1.9-6.0$ & $3.5-5.0$ & $2.5-6.0$ & $3.5-5.0$ \\
\hline $\mathrm{AV} \mathrm{mg} / \mathrm{KOH} / \mathrm{g}$ & 0.21 & 0.087 & 0.37 & 0.40 & 0.50 & 0.05 & 0.80 & 0.50 & 0.50 \\
\hline CC $10 \%$ dat $\%$ mass & 0.26 & 0.11 & 0.38 & 0.39 & 0.30 & 0.05 & 0.30 & 0.05 & 0.30 \\
\hline $\mathrm{H}_{2} \mathrm{O} \&$ sed $\%$ Vol & 0.0 & 0.0 & 0.0 & 0.0 & $500 \mathrm{ppm}$ & $0.05 \%$ & & & \\
\hline Ash \% mass & 0.02 & 0.017 & 0.037 & 0.038 & 0.020 & 0.020 & 0.200 & 0.020 & 0.020 \\
\hline $\mathrm{Na}_{\mathbf{a}}+\mathbf{K} \mathbf{p p m}$ & 2.67 & 1.89 & 4.83 & 4.85 & 5 & 5 & & to report & \\
\hline $\mathrm{Mg}+\mathrm{Cappm}$ & 0.678 & 0.266 & 1.064 & 1.041 & 5 & 5 & 5 & to report & 5 \\
\hline $\mathrm{Al}$ ppm & 0.12 & 0.036 & 0.56 & 0.43 & . & . & - & . & - \\
\hline $\mathrm{Fe}$ & 0.051 & 0.124 & 0.129 & 0.293 & . & . & . & . & . \\
\hline $\mathrm{Zn}$ & 0.073 & 0.115 & 0.10 & 0.255 & . & . & . & . & . \\
\hline $\mathrm{Pb}$ & 0.098 & 0.042 & 0.11 & 0.09 & . & . & . & . & . \\
\hline $\mathrm{Cu}$ & 0.14 & 0.18 & 0.58 & 0.43 & . & . & . & . & . \\
\hline $\mathrm{Ni}$ & 0.3 & 0.28 & 0.67 & 0.66 & 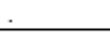 & . & . & . & . \\
\hline
\end{tabular}

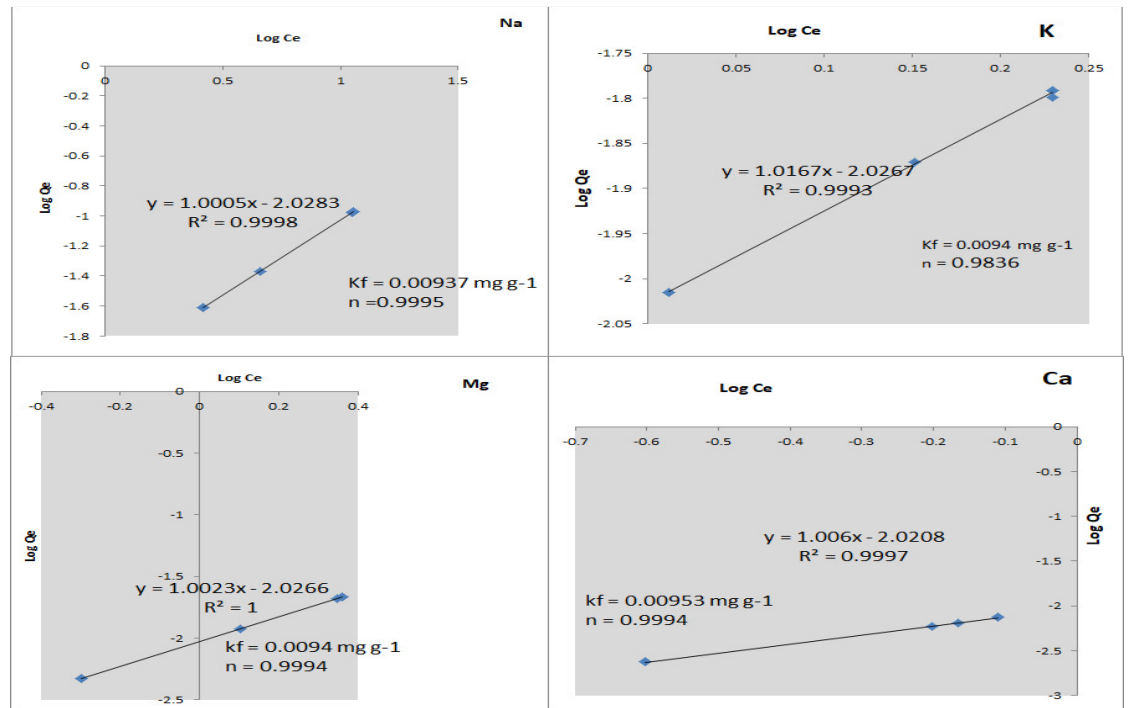

Fig 1: Freundlich isotherm model fit for removal of $\mathrm{Na}^{+}, \mathrm{K}^{2+}, \mathrm{Mg}^{2+}$, and $\mathrm{Ca}^{2+}$ by WPP from produced FAME at equilibrium. WPP has low adsorptive capacity for the metals in FAME and also the adsorption process is unfavourable as depicted by low $k f$ and $n>1$, respectively, and $r^{2}>0.99$.

Pre-treatment of WVOs to generate clean biodiesel has been previously carried out using adsorption process with various adsorbents, such as activated carbon, bleaching earth; and similar to the present study, clean FAME close to biodiesel were obtained from frying oil using silica gel adsorption as a post-transesterification treatment (Kheang et al., 2006; Predojević and Škrbis, 2009; Asri et al., 2015). More recently, same present research group achieved quality ethyl esters using silica gel adsorption as a post treatment following acidbase transesterification of used palm and vegetable oil in ethanolysis process (In Press) though, of lesser yield and quality when compared to the present obtained FAME. As previously stated, metallic content of diesels were not considered. The present WPP adsorption is equally a promising posttransesterification treatment to adsorbed metals and achieves good biofuel properties.

Cluster analysis of $\mathrm{NPO}, \mathrm{NPeO}, \mathrm{NSPO}, \mathrm{NSPeO}$ and NPME, NPeME, NSPME, NSPeME before and after WPP treatment: Principal component analysis using DARwin software analysis package (Perrier and Jacquemoud-Collet, 2006) generated five principal axes that accounted for $99.63 \%$ GD among the feedstocks and their FAME. PCA 1 and PCA 2 contributed a proportion of 63.09 and $27.42 \%$, respectively, of the total variation observed. 


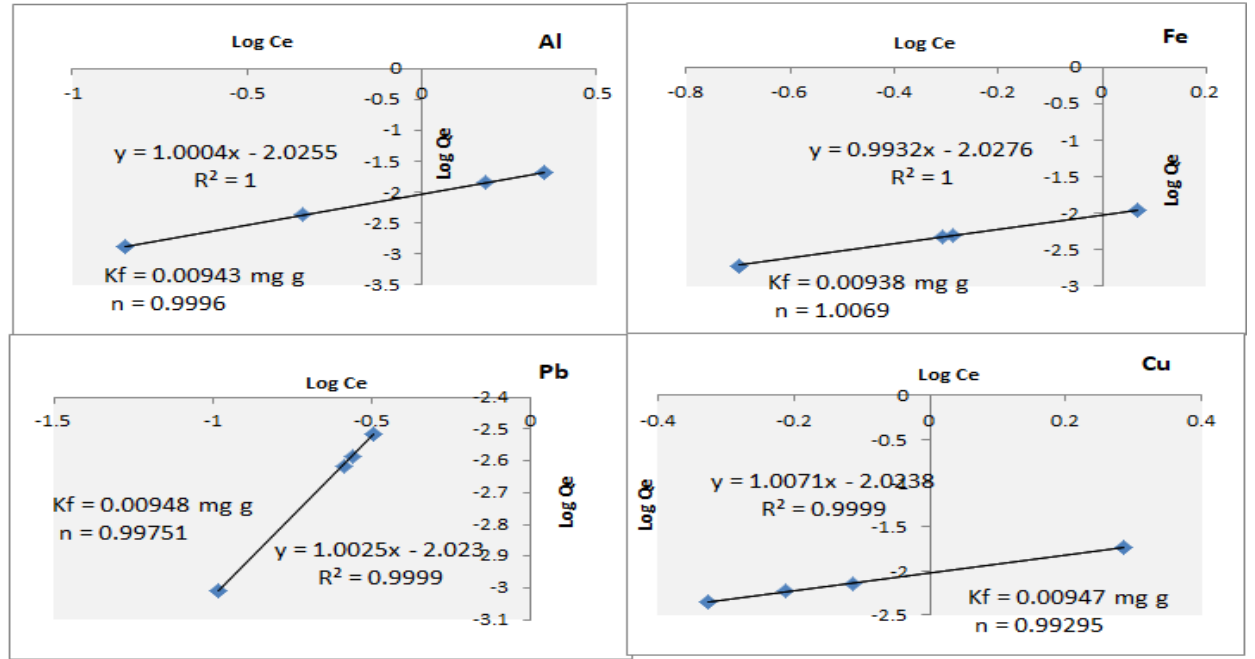

Fig 2: Freundlich isotherm model fit for removal of $\mathrm{Al}^{3+} \mathrm{Fe}^{2+}, \mathrm{Pb}^{2+}$, and $\mathrm{Cu}^{2+}$ by WPP from produced FAME at equilibrium. The adsorption process is unfavourable as depicted by low $k f$ and $n>1$, respectively, and $r^{2}>0.99$, except for $\mathrm{Fe}^{2+}$

The two dimensional scatter plot of PCA 1 vs PCA 2 (Figure 2) demarcates the feedstocks and FAME into four quadrants of three clusters. The vegetable oil feedstocks were found at the upper left quadrants as cluster 1, characterized with high viscosity, FP, AV, $\mathrm{CC}$ and metal content, indicating them as non-fit biofuel for modern diesel engine (Bart et al., 2010; Giakoumis et al., 2013) and two steps transesterified VOs are at upper right quadrant as cluster 2 and were further differentiated with respect to feedstock source and transesterification step number. The overlapping of NSPME and NSPeME in each case, depict them as of equal quality while NPME and NPeME are with short distances, specifying higher Group I and II metal content of NPME over NPeME. The cluster 2 FAMEs have good biofuel properties but their metallic content is undesirable (Lobo et al., 2011). Cluster 3 contains finalized FAMEs obtained with WPP adsorption-post two steps alkali-transesterification treatment and they clearly distinguished themselves at the lower left quadrant as neat biodiesel with desirable biofuel properties and low susceptibility of combustion chambers to corrosion by group I and II metals. According to principal components analysis using SAS, the main biofuel properties that contributed substantially to the observed demarcations among the feedstocks and FAME are viscosity, CC, AV, ash, Na, $\mathrm{Mg}, \mathrm{Ca}, \mathrm{Al}, \mathrm{Cu}$ and $\mathrm{Ni}$. The present cluster groupings show visualized disparity in characteristics of assessed biofuels, though, scantly use, is useful for quality characterization of feedstocks, FAME and or biodiesel.

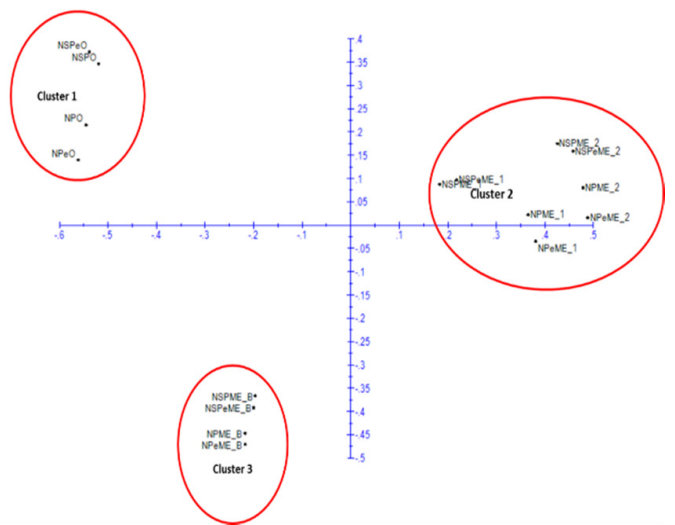

Fig 3: A scatter plot of feedstock and FAME before and after post-transesterification treatment with WPP following two steps transesterification processes as generated using PCA in DARwin software package. _l, at 
single step transesterifiction; _2, two steps ransesterifcationa and _B, adsorption with WPP as posttransesterification treatment.

Conclusions: The present study shows that adsorption of FAME with WPP as post-transesterification treatment was able to reduce the metal content, AV and $\mathrm{CC}$ into acceptable limit across diverse International standards. Though, the adsorption process using WPP was cooperative with low adsorption capacity, but WPP showed good potential as locally available and cheap adsorbent for purifying FAME to desirable quality and is amenable to improvement. The obtained results in Part I and II of these studies point towards a sustainable process for revalue and reuse of low valued SpVOs into production of high valued biodiesel.

\section{REFERENCES}

Altin, R; Çentinkaya, S; Yucesu, HS (2001). The potential of using vegetable oil fuels as fuel for diesel engines. En. Conv. Managm. 42: 529-538.

Asri, NP; Sari, ADP; Poedjojono, B; Suprapto, S (2015). Pre-treatment of waste frying oil for biodiesel production. Modern Applied Science 9(2):99-106

Bakir, ET; Fadhil, AB (2011). Production of biodiesel from chicken frying oil. Pak. J. Anal. Environ. Chem. 12 (1\& 2): 95-108.

Bart, JCR; Palmeri, N; Cavallaro, S (2010). Biodiesel Science and Technology- From soil to oil, $7^{\text {th }}$ Series, Wood Head Publishing limited, Cambridge CB21 6AH UK. 2010.

Canakci, M (2007). The potential of restaurant waste lipids as biodiesel feedstocks. Bioresour. Technol. 98: 183-190.

Cvengroš, J; Cvengrošová, Z (2004). Used frying oil and fats and their utilization in the production of methyl esters of higher fatty acids. Biomass Bioen. 27:173181.

de Araújo CDM; de Andrade CC; de Silva ES; Dupas FA (2013) Biodiesel production from used cooking oil: a review. Renew. Sustain. Energy Rev. 27:445-452.

Encinar, JM; Gonzále, JF; Rodriguez-Reinares, A (2005). Biodiesel from used frying oil: Variables affecting the yields and characteristics of the biodiesel. I EC Res. 44: 5401-5499

Felizardo, PM; Correia, J; Raposo, I; Mendes, JF; Berkemeir, R; Boedado, JM (2006). Production of biodiesel from waste frying oils. Waste Manag. 26: 487-494.

Filho, SCS; Silva, TAF; Miranda, AC; Fernandesa, MPB; Felico, HH; Calarge, FA; Santana, JCC; Tambourgi,
EB (2014). The potential of biodiesel production from frying oil used in the restaurants of São Paulo City. Brazil. CET 37: 577-582

Giakoumis, EG (2013). A statistical investigation of biodiesel physical and chemical properties, and their correlation with the degree of unsaturation. Renewable Energy 50: 858-878.

Kheang, LH; May, CY; Foon, CS; Ngan, MA (2006). Recovery and conversion of palm olein-derived used frying oil to methyl esters for biodiesel. Journal of Oil Palm Research 18:247-252.

Kumar, U; Bandyopadhyay, M (2006). Sorption of cadmium from aqueous solution using retreated rice husk, Bioresour. Technol. 97: 104-109

Leung, DYC; Chen, GY (2000). Biodiesel production using waste cooking oil from restaurants, In: Cheng $\mathrm{P}$ (ed) Energy Engineering in the $21^{\text {st }}$ Century, Vol. 4 Begell House Inc., Hong-Kong, New York, p, 1553

Lobo, FA; Gouvaveia, D; Oliveira, AP; Romão, PC; Fraceto, LF; Dias, NL; Ros, AH (2011). Development of a method to determine $\mathrm{Ni}$ and $\mathrm{Cd}$ in biodiesel by graphite furnace atomic absorption spectrometry. Fuel 90 (1): 142-146

Moyib, OK; Ayedun, MA; Awokoya, OJ; Omotola, OE (2017). Waste printing paper as analogous adsorbents for heavy metals in aqueous solution. NJCR 22(1): 33-44.

Perrier, X; Jacquemoud-Collet, JP (2006). DARwin software. http://darwin.cirad.fr/darwin.

Predojević, ZJ; Škrbis, BD (2009). Alkali-catalyzed production of biodiesel from waste frying oils. $J$. Serb. Che. Soc. 74(8 \& 9): 993-1007.

Proctor, A; Toro-Vanzquez, JF (2009). The Freundlich isotherms in studying adsorption in oil processing. In: List $\mathrm{G}$ (ed) Bleaching and purifying fats and oilsTheory and Practice, AOAC, Elsevier Inc., UK, p.209.

Roh, H; Kim, S; Jung, J (2014), adsorption of heavy metal ions lead (ii) and copper (ii) on perm- lotion- treated human hair. Korean J. Chem. Eng. 31(2): 310- 314.

Statistical analysis system (SAS) (2002) Version 8. SAS Institute Incorporation, NC Cary. 